$15^{\text {th }}$ International Conference on

AEROSPACE SCIENCES \& AVIATION TECHNOLOGY,

ASAT - 15 - May 28 - 30, 2013, Email: asat@mtc.edu.eg,

Military Technical College, Kobry Elkobbah, Cairo, Egypt,

Tel: +(202) 24025292 -24036138, Fax: +(202) 22621908

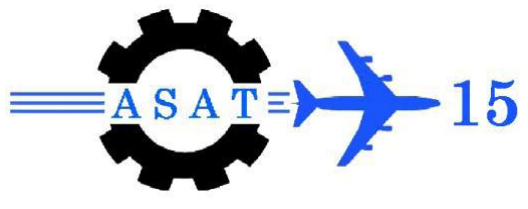

\title{
Enhancement of Stopband Attenuation Bandpass Filter Using Complementary Split Ring Metamaterial Resonators
}

\author{
$\left\{\text { M. A. Fouad*, M. A. Abdalla }{ }^{\dagger}, \text { H. A. Elregeily, A. A. Metkess }\right\}^{\dagger}$
}

\begin{abstract}
In this paper, a simple parallel-coupled band pass filter with a metamaterial complementary split ring resonator is introduced for improvement of the filter stopband attenuation. The theoretical concept of the complementary split ring resonator is introduced. The designed filter has been examined theoretically using circuit modelling and electromagnetic full wave simulation. The results demonstrate that the reported filter can enhance the stopband attenuation three times compared to conventional filter with maintaining typical linear phase response and constant group delay. Moreover, these results have been obtained using only one unit cell for the sake of filter compactness and simplification.
\end{abstract}

Keywords: Metamaterial, complementary split ring resonator, CSRR, Filter, periodic structure.

\section{Introduction}

The electric permittivity $(\varepsilon)$ and magnetic permeability $(\mu)$ of a medium are the electromagnetic constitutive parameters of a medium which are used to describe the material behaviour on applying an electromagnetic wave. The definitions of these parameters depend on the electromagnetic interactions with the structure. In general, metamaterial artificial effective medium are electromagnetic ordered array scatters satisfying a sufficient long wavelength condition at $\mathrm{RF} /$ microwave frequencies to demonstrate particular electromagnetic properties. This concept has been expanded to demonstrate different metamaterials types. These include the negative permeability metamaterials, the negative permittivity metamaterials, and their simultaneous negative permittivity and permeability. In general, metamaterials can be defined as artificially constructed materials having electromagnetic properties that are generally not found using conventional, naturally occurring materials.

A negative permittivity medium has an imaginary propagation constant such that no wave can propagate within such medium and hence an incident electromagnetic wave on this medium will be reflected back. In nature, plasma is a medium observed to have a negative permittivity. The plasma has a dispersive permittivity such that below certain frequency called the plasma frequency $\left(\omega_{\mathrm{p}}\right)$, the medium permittivity is negative. In 1996, Pendry [1,2] introduced artificial electrical plasma consisting of a three dimensional array of intersecting thin straight wires. The electrical plasma frequency is dependent on the wire radius and spacing and hence it is possible to design it to be in the microwave band [1]. On the other hand, in nature, it has been explained that negative effective dispersive permeability, with some losses, can be

\footnotetext{
m.a.fouadhagag@ieee.org

$\dagger$ maaabdalla@ieee.org

Egyptian Armed Forces, Egypt.
} 
possible in some certain insulating ferromagnets [3]. In 1999, Pendry proposed an artificial medium consisting of nonmagnetic conducting materials that could exhibit a large response to the magnetic field of electromagnetic wave [4]. This model was based on an array of circular split ring resonators (SRRs) structure. Complementary split ring resonator (CSRR) is characterized with an equivalent negative permittivity and hence it has been introduced as a metamaterial structure [5]. Thanks to the novel properties of electromagnetic waves propagation in metamaterials, their response has had a huge effect in various applications on physics, optics, guided, radiated, and refracted wave applications [6,7]

Parallel-coupled, half wavelength resonator filters are positioned so that adjacent resonators are parallel to each other along half of their length. This parallel arrangement gives relatively large coupling for a given spacing between resonators, and thus, this filter structure is particularly convenient for constructing filters having a wide bandwidth [8]. However, such filters may suffer from not excellent stopband attenuations. In this paper, we introduce the use of complementary split ring resonator for improvements of the stopband attenuations of coupled parallel coupled half wave resonator microstrip filters. The employed CSRR was etched on the ground plane between conducting strips of the coupled line filter. Only one CSRR unit cell was used for the sake of a simple filter design.

\section{CSRR Theory}

It has been suggested that the use of periodic array of split ring resonator excited by a magnetic field parallel to ring axis is able to introduce an artificial negative permeability metamaterial that may be implemented as a three dimensional periodic structure [2]. However, the $\mathrm{H}$-field excitation provided by the incident field on conventional split ring resonator is not strong enough to produce a noticeable effect. Thus, a multilayered architecture is required to produce a sufficient response. Therefore, in summary, we can say that use of SRR is not convenient with microstrip configuration.

On the other hand, complementary split spring resonator, excited by an electric field polarized in the axial direction of the ring so that it results in negative permittivity, was introduced to overcome this problem [5]. From electromagnetic theory, if the thickness of the metal plates is zero and the conductivity it exhibits is infinite then the apertures behave as perfect magnetic conductors and hence the CSRR behaviour can be considered as a dual (or complementary) of SRR. Therefore, CSRR can be easily fabricated using microstrip configuration by etching the rings in the ground plane beneath the upper microstrip transmission strip.

From circuit point of view, the CSRR particle can be described as a parallel resonant tank with inductance $\mathrm{L}_{c}$ and capacitance $\mathrm{C}_{c}$. The CSRR particle behaves like an electric dipole which is excited by an external axial electric field (in this case the electric field of the microstrip line). $\mathrm{L}$ and $\mathrm{C}$ represents the per unit length inductance and capacitance of the host line which is electrically coupled to the CSRR. The lumped element equivalent circuit model for the CSRR loaded transmission line is depicted in Fig. 1. The analysis of the CSRR equivalent circuit should represent a negative permittivity metamaterial structure whose bandwidth is approximately cantered at the resonant tank circuit whose resonant frequency $\left(\mathrm{f}_{0}\right)$ can be approximated as: 


$$
f_{0}=\frac{1}{2 \pi \sqrt{L_{c} C_{c}}}
$$

It is important to note that the resonance frequency is too sensitive to the coupling between the couplings between the two rings. The exact resonant frequency can be obtained using good parametric analysis for the employed CSRR which is out of scope of our paper. However, equation (1) is still considered acceptable approximation for the initial design of the CSRR unit cell resonance frequency and hence its associated negative permittivity frequency band in the initial design stage of CSRR loading a microstrip transmission line.

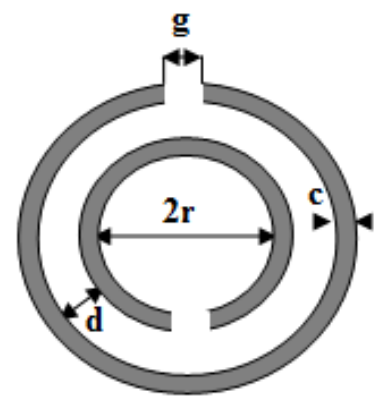

(a)

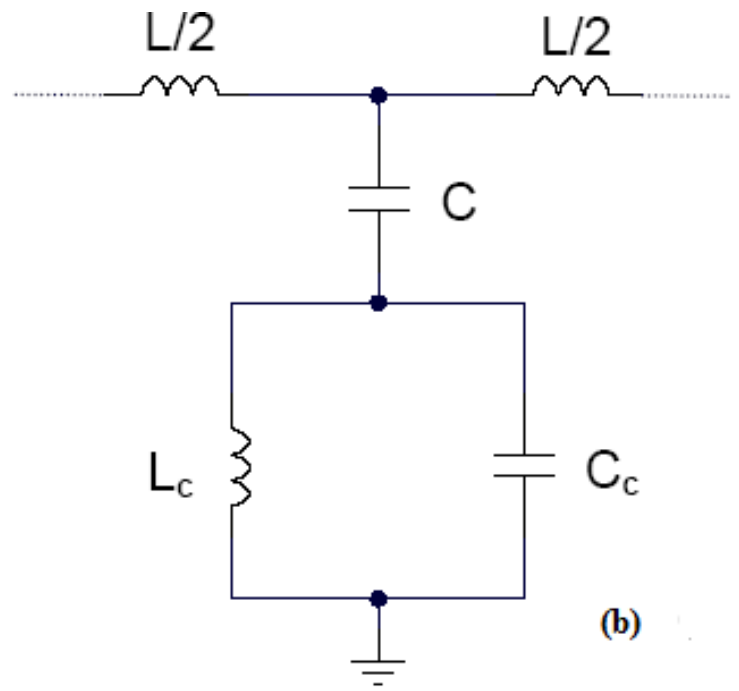

Fig. 1 (a) The layout of the microstrip single CSRR particle (b) The lumped-elements equivalent circuit of the CSRR

\section{Structure and Design}

In this section we introduce the problem of slow attenuation of coupled line filter and the design of metamaterial CSRR structure to enhance the performance of the coupled line filter without the need to increase the filter order. The design of the proposed filter using metamaterial CSRR was done using the described modelling circuit for the metamaterial CSRR particle in Fig. 1. Moreover, the design was validated using the electromagnetic full wave analysis. The commercial finite element software ANSOFT-HFSS was employed.

The layout of the Parallel-coupled, half-wavelength resonator band-pass filter along with CSRR is shown in Fig. 2. A Rogers RO3006(tm) with dielectric constant, $\varepsilon_{\mathrm{r}}=6.15$ and height, $\mathrm{h}=1.6 \mathrm{~mm}$ was employed. The detailed filter dimensions of the introduced filter are shown in figure. The structure is symmetric and the separation between the wider lines $s 1=15$ mil, thinner lines s $2=12 \mathrm{mil}$, and the width of the thinner line $\mathrm{w} 3=0.889 \mathrm{~mm}$. The overall filter length is $54.85 \mathrm{~mm}$ while the filter width is $49.5 \mathrm{~mm}$. The filter is designed using design equations in [9]. 


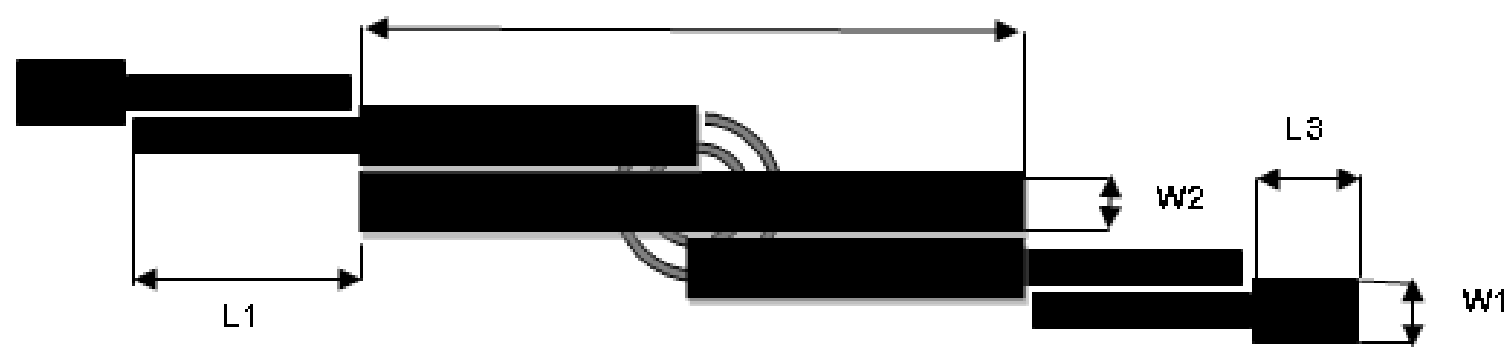

Fig. 2 The layout of parallel-coupled, half-wavelength resonator band-pass filter $\left(\mathrm{L}_{1}=11.39 \mathrm{~mm}, \mathrm{~L}_{2}=21.59 \mathrm{~mm}, \mathrm{~L}_{3}=5 \mathrm{~mm}, \mathrm{~W}_{1}=2.4 \mathrm{~mm}\right.$, $\mathrm{W}_{2}=1.9 \mathrm{~mm}$, and $\mathrm{w}_{3}=0.889 \mathrm{~mm}$.)

The frequency response of the filter is shown in Fig.3. As illustrated in the figure, a bandpass filter operates from $2.6 \mathrm{GHz}$ to $3.8 \mathrm{GHz}$; i.e $3.2 \mathrm{GHz}$ centre frequency with $37.5 \%$ fractional bandwidth. Also, the filter has upper stopband with a $20 \mathrm{~dB}$ upper stopband attenuations within $600 \mathrm{MHz}$.

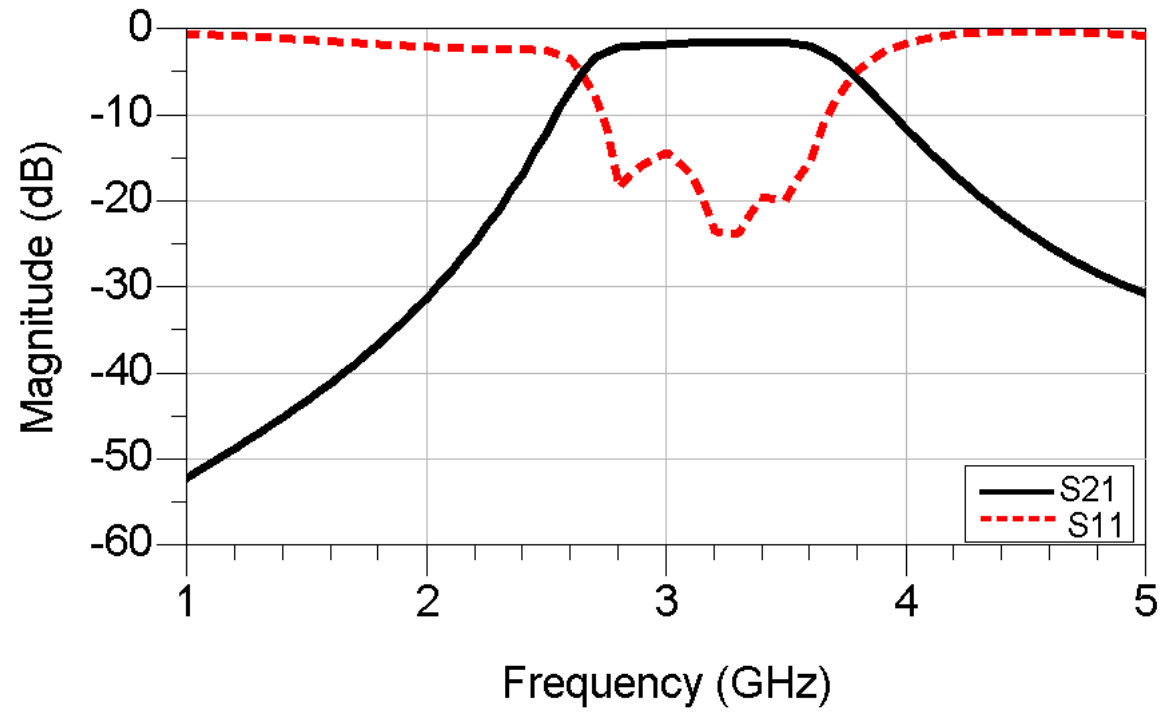

Fig. 3 The scattering parameter magnitudes of conventional parallel-coupled filter (without CSRR)

To enhance the previous filter performance in terms of increasing the increasing filter stopband attenuation, it is cascaded with a metamaterial stopband metamaterial CSRR filter etched on the ground plane. For the sake of filter compactness, only one CSRR unit cell was employed. The metamaterial CSRR loading a microstrip transmission line structure is shown in Fig. 4. As shown in the figure, the used CSRR particle was cell formed by two concentric circular CSRR was etched in the centre of the ground plane. The basic unit cell of a CSRR loaded transmission line has an inner diameter of $3.6 \mathrm{~mm}$, two ring separation of $0.3 \mathrm{~mm}$, and ring width of $0.3 \mathrm{~mm}$.

The design objective of the metamaterial CSRR to demonstrate its negative permittivity frequency band approximately around $4 \mathrm{GHz}$ with a bandwidth sufficient enough to overlap with the coupled line filter attenuation stopband. This design objective was examined by studying the scattering parameters of the microstrip transmission line loaded with a metamaterial CSRR particle etched on the ground plane by both circuit modelling (shown in 
Fig. 1) and electromagnetic full wave simulation using finite element technique. The commercial HFSS is used for this purpose.

The design results obtained using circuit model and HFSS simulations are shown in Fig. 5. As shown in the figure, there is a reasonable agreement between the circuit model design and full wave simulations. Both results indicate that the metamaterial CSRR filter has a stopband in frequency band $3.7 \mathrm{GHz}-4.3 \mathrm{GHz}$. This stopband can be explained due to the negative effective permittivity obtained as a result of the etching of the CSRR. As explained above, the negative permittivity frequency band can be approximately centred at the resonance frequency of tank circuit shown in the CSRR equivalent circuit shown in Fig. 1.

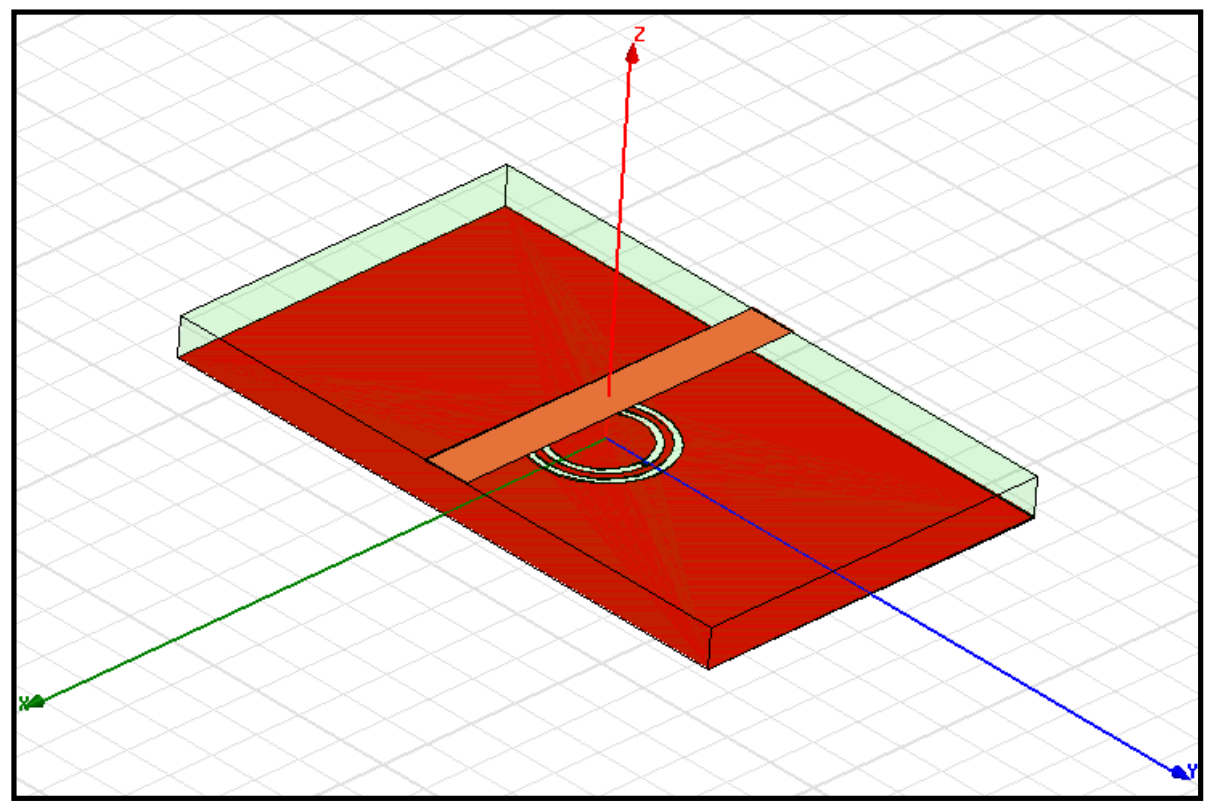

Fig. 4: A microstrip transmission line loaded with a metamaterial CSRR etched on ground plane.

For the sake of confirmation, the equivalent circuit elements of the tank circuit were extracted to be as follow, $\mathrm{C}_{\mathrm{c}}=2.3 \mathrm{pF}, \mathrm{L}_{\mathrm{c}}=0.6 \mathrm{nH}, \mathrm{L}=1.7 \mathrm{nH}$, and $\mathrm{C}=-.6 \mathrm{pF}$. Hence, the resonance frequency can be calculated using (1) to be as $4.3 \mathrm{GHz}$ which is a little bit higher that the stopband obtained in the both circuit/HFSS simulation results shown in Fig. 5. This difference is due to the simplification in describing the resonance frequency of CSRR particle using (1). However, as explained earlier, this frequency can be acceptable for initial design purpose. More accurate resonance frequency of the CSRR particle loading a microstrip transmission line can be obtained using intense parametric analysis which was out of our manuscript scope.

\section{Result and Analysis}

In this section we introduce the results for using the CSRR element to improve the upper stopband attenuation of the coupled line band pass filter whose response is shown in Fig. 2 . This was done by cascading that coupled line band pass filter with the metamaterial CSRR filter whose response is shown in Fig. 4. It is worth to mention that some fine optimization has been done for CSRR position. Finally, the proposed filter structure is shown in Fig. 6.

The simulated scattering parameters for the filter with CSRR are shown in Fig.7. As shown, the filter can operate within the same frequency band from almost $2.6 \mathrm{GHz}$ to $3.8 \mathrm{GHz}$, the 
same frequency band illustrated in Fig. 2. However, there is significant improvement in the upper stopband attenuation. It is obvious that the filter has a $20 \mathrm{~dB}$ upper stopband attenuations within $200 \mathrm{MHz}$. Compared to conventional coupled line filter response in Fig. 2, the $20 \mathrm{~dB}$ attenuation was $600 \mathrm{MHz}$. Therefore, we can claim that the metamaterial can introduce three times enhancement of the $20 \mathrm{~dB}$ attenuation compared to the conventional coupled line filter; without the use of the CSRR.

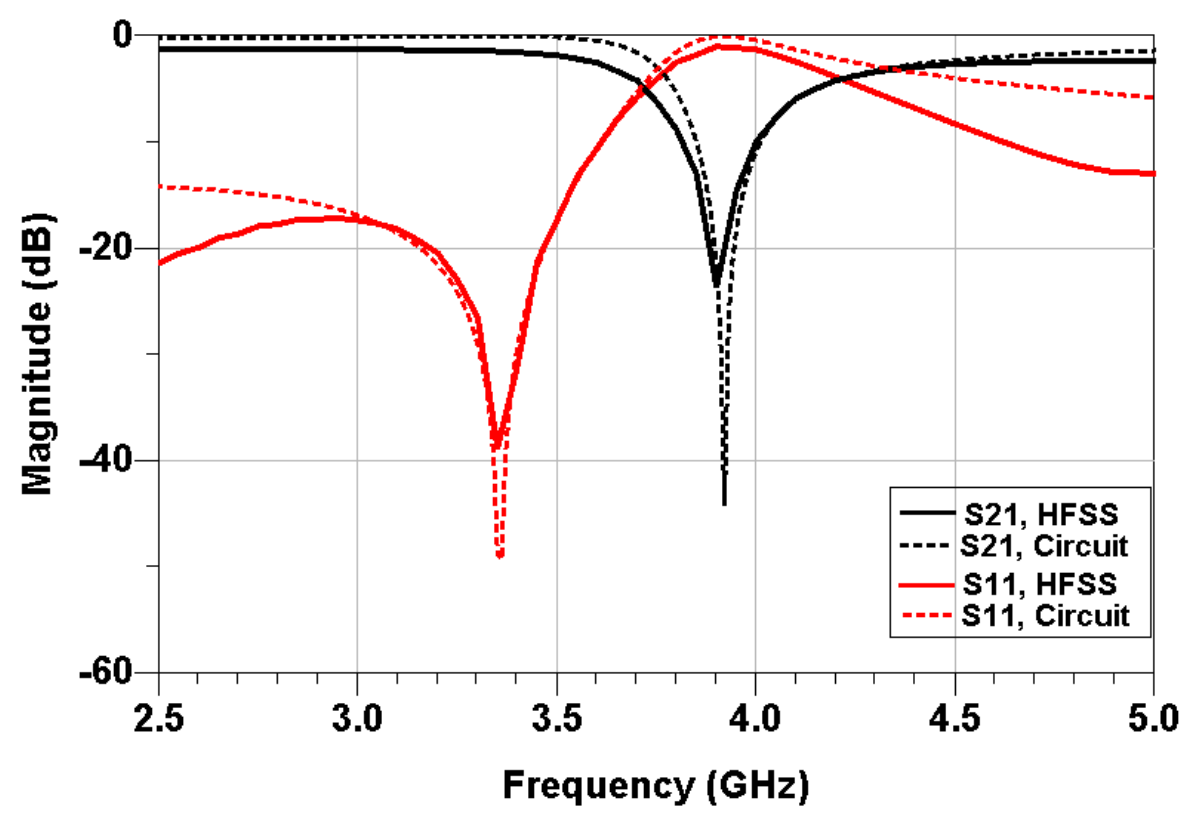

Fig. 5 The scattering parameter magnitudes of a microstrip transmission line loaded with a metamaterial CSRR filter etched on ground plane

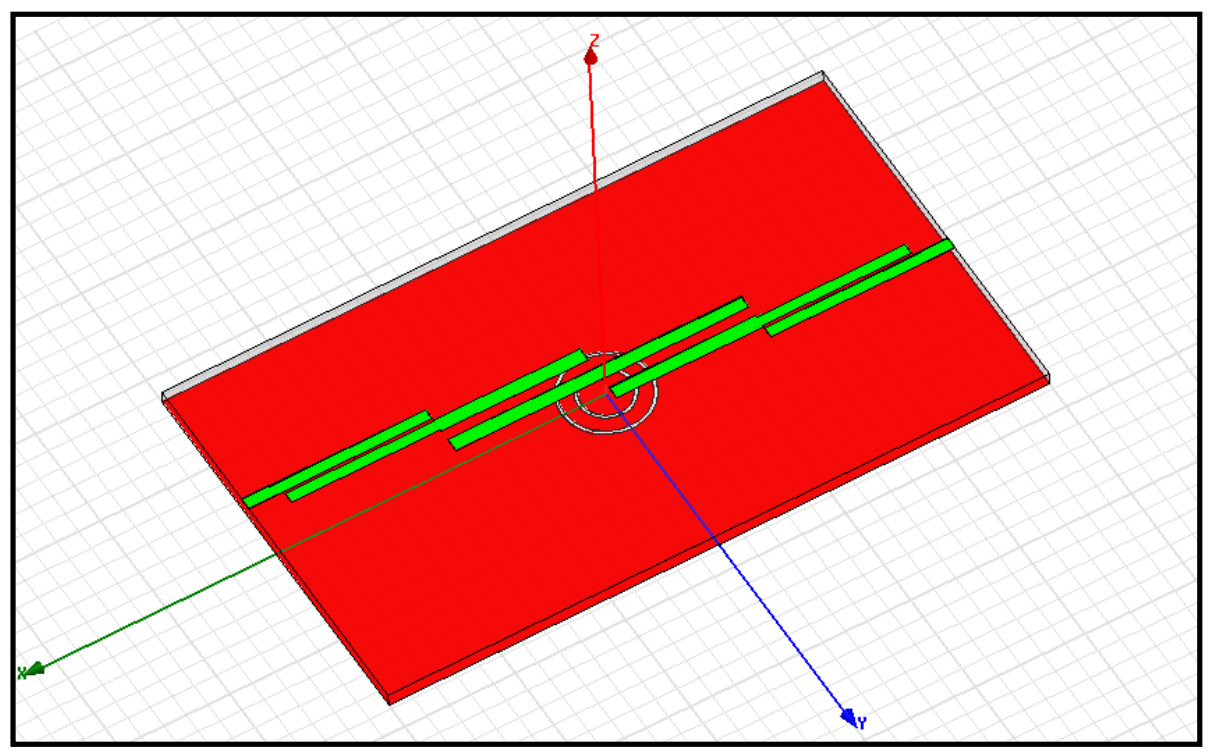

Fig. 6 The 3D structure of the coupled line filter cascaded with the ground etched metamaterial CSRR filter. 


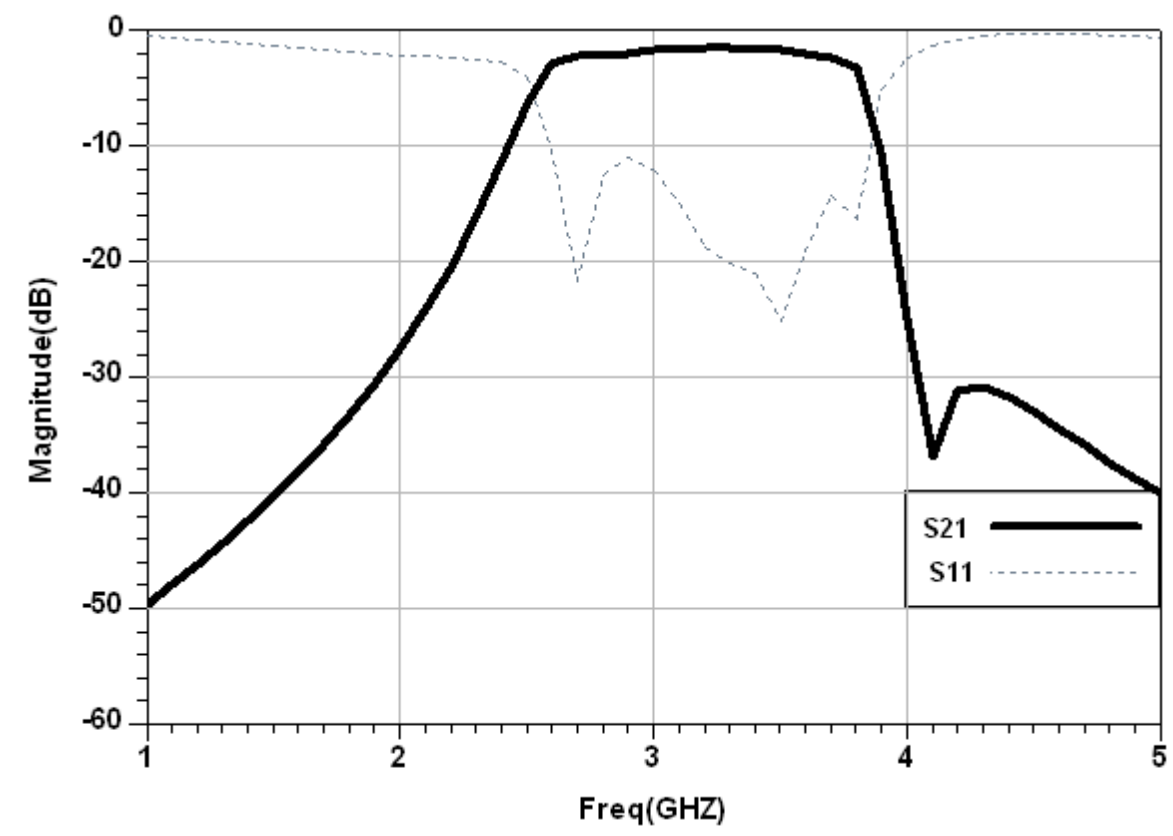

Fig. 7 The scattering parameter magnitudes of parallel-coupled filter (with CSRR).

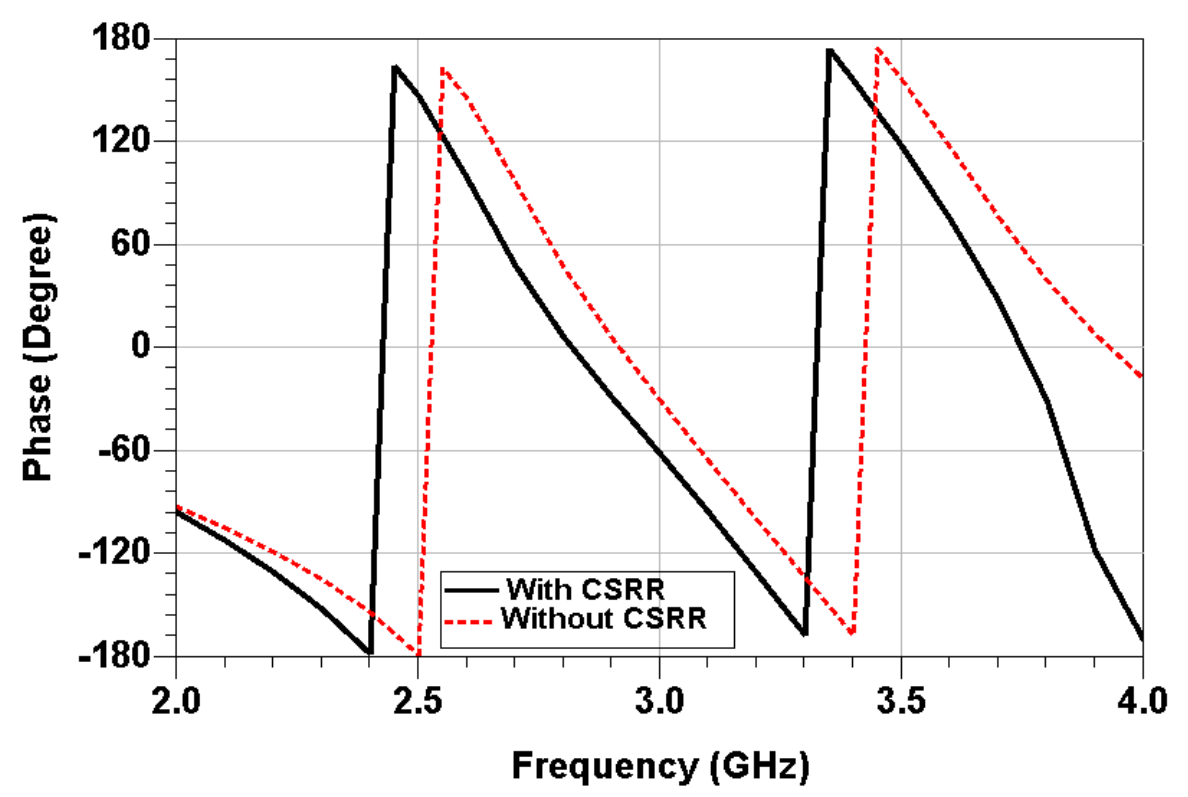

Fig. 8 The phase of scattering parameter $\left(S_{21}\right)$ of the proposed parallel-coupled filter (with CSRR) and conventional filter (without CSRR).

To confirm that the use of the CSRR does not affect the conventional coupled line filter response, the phase response of the scattering parameter $\left(S_{21}\right)$ is studied and compared to the phase response of the conventional coupled line filter. Both results are depicted in Fig. 8. As shown, the use of the CSRR along with the filter keeps the filter typical linear phase response within its frequency passband $(2.6 \mathrm{GHz}-3.8 \mathrm{GHz})$. However, there is some phase differences between the two studied cases. This can be explained better by focusing the group delay of the two filter cases. This new comparison is plotted in Fig. 9. As shown in the figure, the proposed filter along with CSRR has almost constant group delay over the filter passband. Compared to the conventional coupled line filter, we can claim that the group delay of the proposed filter with the CSRR group delay is still almost constant. 


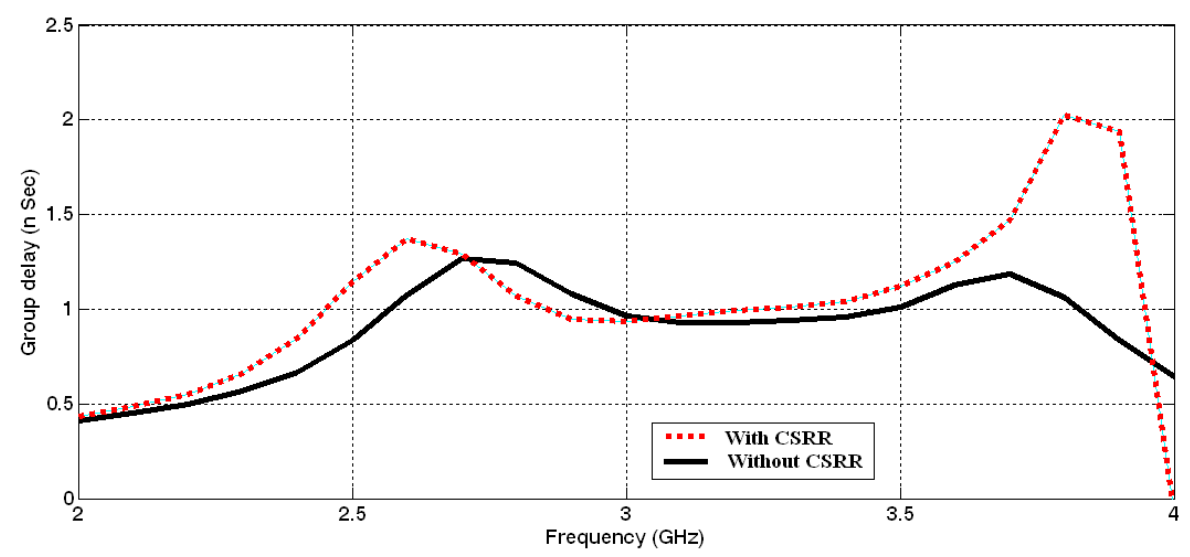

Fig. 9 The group delay of scattering parameter $\left(S_{21}\right)$ of the proposed parallel-coupled filter (with CSRR) and conventional filter (without CSRR).

\section{Conclusion}

A simple and compact coupled line passband filter cascaded with a metamaterial CSRR filter has been presented. The introduced filter improves the filter roll-off factor without the need to increase the filter order. The filter design has been introduced using circuit model and verified using the electromagnetic full wave analysis. The results indicate that the proposed modified coupled line filter has three times better roll off factor at its upper stopband. This modified and simple filter can become a powerful tool for several filter applications, examples: in devices such as image rejection filters, antenna duplexer-filters, and filters of wireless communication application.

\section{References}

[1] Pendry, J. B., Holden, A. J., Robbins, D. J., and Stewart, W. J., "Low frequency plasmons in thin-wire structures," Journal of Physics: Condensed Matter, vol. 10, pp. 4785-809, 1998.

[2] Pendry, J. B., Holden, A. J., Stewart, W. J., and Youngs, I., "Extremely low frequency plasmons in metallic mesostructures," Physical Review Letters, vol. 76, pp. 4773-6, 1996.

[3] Hartstein, A., Burstein, E., Maradudin, A. A., Brewer, R., and Wallis, R. F., "Surface polaritons on semi-infinite gyromagnetic media," Journal of Physics C (Solid State Physics), vol. 6, pp. 1266-76, 1973.

[4] Pendry, J. B., Holden, A. J., Robbins, D. J., and Stewart, W. J. "Magnetism from conductors and enhanced nonlinear phenomena," IEEE Transactions on Microwave Theory and Techniques, vol. 47, pp. 2075-84, 1999.

[5] Falcone, F., Lopetegi ,T., Baena, J. D., Marques, R., Martin, F., and Sorolla, M., "Effective negative- \&epsilon; stopband microstrip lines based on complementary split ring resonators," IEEE Microwave and Wireless Components Letters, vol. 14, pp. 280282, 2004.

[6] Eleftheriades, G. V. and Balmain, K. G., Negative refractive metamaterials. New jersey: John Wiey \& Sons, 2005.

[7] Caloz, C., Itoh, T., Electromagnetic Metamaterials: Transmission Line Theory and Microwave Applications, John Wiley \& Sons, Inc., 2006.

[8] Mattaei, G., Young, L., and Jones, E. M. T., Microwave Filters, Impedance-Matching Networks, and Coupling Structures, Artech House, Norwood, MA, 1980.

[9] Pozar, D.M., Microwave Engineering, $3^{\text {rd }}$. ed., New York: J. Wiley \& Sons, 2005. 